Recibido:

28-IV-2020

Aceptado:

$18-\mathrm{V}-2020$

Publicado en línea:

25-V-2020
Determinantes de la calidad de vida relacionada con la salud oral en adultos mayores de un centro diurno costarricense: Resultados de un estudio piloto

\section{Oral Health-Related Quality of Life Determinants in the Elderly from a Costa Rican Day Center: Findings of a Pilot Study}

Cristina Barboza-Solís DDS, MSc, PhD¹; María José Araya-Jiménez;

Sonia Stradi-Granados DDS, MAG'1; Juan Barahona-Cubillo DDS;

Kattyusffka García-Aguirre ${ }^{1}$, Carolina Carranza-Chacón ${ }^{1}$

1. Facultad de Odontología, Universidad de Costa Rica, Costa Rica.

Autor para correspondencia: Dra. Cristina Barboza-Solís - cristina.barbozasolis@ucr.ac.cr

RESUMEN: El cambio de la distribución demográfica en Costa Rica establece a las personas adultas mayores (PAM) como una prioridad de salud pública. La salud oral es un indicador multidimensional, que incluye dimensiones biológicas, sociales y psicológicas. En este campo de investigación, además de utilizar las medidas de morbi-mortalidad, se ha dado una importancia a distintos indicadores que pretenden aproximar otras dimensiones subjetivas. Dentro de estas, la calidad de vida toma cada vez más importancia. Este estudio pretende identificar los principales determinantes de la Calidad de Vida Relacionada con la Salud Oral (CVRSO) en PAM de un centro diurno del cantón de Desamparados en San José, Costa Rica. Se trata de un estudio descriptivo transversal de tipo observacional realizado entre 2018 y 2019. La CVRSO, se midió a través del instrumento GOHAI (General/Geriatric Oral Health Assesment Index). Como variables independientes se incluyeron las sociodemográficas, socioeconómicas, de morbilidad, comportamientos de salud y consumo de medicamentos. Se realizaron análisis bivariados con las pruebas no paramétricas de Wilcoxon, Kruskall-Wallis y Spearman, utilizando el paquete estadístico STATA 14. Este estudio revela que existen diferencias de promedio al comparar la CVRSO y los determinantes demográficos, socioeconómicos, de morbilidad, toma de medicamentos y comportamientos de salud. Los mayores de 80 años, las mujeres, aquellos con niveles superiores de escolaridad, de ingresos altos, sin edentulismo, con niveles bajos de xerostomía, sin enfermedades, que no toman medicamentos, que no fuman, hacen deporte, que no meriendan y que consumen moderadamente azúcares, son aquellos que reportan una mejor CVRSO comparados a sus contrapartes. 
PALABRAS CLAVE: Calidad de vida relacionada con la salud oral; Determinantes; GOHAl; Persona adulta mayor; Costa Rica.

ABSTRACT: The change in the demographic distribution of Costa Rica establishes the elderly as a public health priority. Oral health is a multidimensional indicator, which includes biological, social, and psychological dimensions. Besides using measures of morbidity and mortality, different indicators seek to approximate other subjective dimensions. There is an increasing interest in analyzing the role of quality of life on health. This study aims to identify the main determinants of Oral Health-Related Quality of Life (OHRQL) in the elderly attending a day center in the city of Desamparados (San José, Costa Rica). This is a descriptive cross-sectional study ran between 2018 and 2019. OHRQL was measured via the General / Geriatric Oral Health Assessment Index (GOHAl). As independent variables, sociodemographic, socioeconomic, morbidity, health behaviors, and drug consumption were included. Bivariate analyzes were performed using the Wilcoxon, Kruskall-Wallis, and Spearman non-parametric tests, using STATA 14. This study revealed differences in GOHAl scores according to demographic, socioeconomic, morbidity, medication, and health behaviors. Those over 80 years old, women, with higher levels of education, high income, without edentulism, with low levels of xerostomia, people without diseases, who do not take medication, who do not smoke, play sports, do not snack and who consume moderately sugars, are those that report a better OHRQL compared to their counterparts.

KEYWORDS: Oral health related quality of life; Determinants; GOHAl; Elderly; Costa Rica.

\section{INTRODUCCIÓN}

Costa Rica enfrenta actualmente uno de los principales retos demográficos de su historia. Entre 1950 y 2018 la población en el territorio se sextuplicó, pasando de 868934 a 5003402 (1). Esta composición demográfica ha sido la que ha establecido las principales bases de organización social de las instituciones públicas, particularmente aquellas relacionadas con los servicios de salud. En 1950, la población de 65 años y más representaba 3.5\% de la población (1). Para el 2018 este porcentaje aumentó a $8.2 \%$ y se estima que para el 2050, este se elevará a 20.2\%, para un total de 1262311 personas adultas mayores (PAM) que habitarán en el país (1). Asimismo, la transición demográfica ha llevado consigo una transición epidemiológica. En el siglo XIX, los principales problemas de salud pública se relacionaban con las enfermedades infecciosas (2). Actualmente, son las enfermedades crónicas, caracterizadas por largo periodos de latencia, las que afectan a la mayor cantidad de población adulta (2).

La salud es un indicador multidimensional, que incluye dimensiones biológicas, sociales y psicológicas (3). Para fines de investigación, se han utilizado tradicionalmente medidas de morbilidad y mortalidad (4), incorporando diversas características biológicas de las enfermedades (3). Sin embargo, dichas medidas son reductoras y ocultan gran parte de la complejidad de los procesos de saludenfermedad, particularmente en algunas poblaciones (5). Recientemente, investigadores han propuesto una aproximación de las dimensiones más subjetivas y psicológicas de la salud, para comprender mejor la complejidad de la construcción de la salud a lo largo del curso de la vida (6). Es desde esta 
perspectiva, que se desarrolla desde hace más de 30 años el estudio del Envejecimiento Sano (o Healthy Ageing en inglés), que pretende describir todos los mecanismos, e identificar los determinantes que pueden influir sobre la salud de las personas, desde su nacimiento, hasta su muerte (6). En este campo de investigación, además de utilizar las medidas de morbi-mortalidad, se ha dado una importancia a distintos indicadores que pretenden aproximar dimensiones subjetivas del bienestar y la calidad de vida de las personas (7).

Dentro de estas, la calidad de vida toma cada vez más importancia. La Calidad de Vida Relacionada con Salud Oral (CVRSO) ha sido definida por Degrandi y colaboradores (2017) como "una valoración multidimensional y auto-reportada, que mide el impacto de las condiciones bucales sobre las actividades de la vida diaria" (7), una medida importante pues el autoestima y el bienestar se pueden ver disminuidos por trastornos de la cavidad bucodental (8).

Es conocido que las enfermedades crónicas son mutifactoriales, y parte de los elementos que juegan un rol en los procesos salud-enfermedad de estas patologías se relacionan con los determinantes sociales (9). Por lo tanto, si se desea analizar la CVRSO, se deben incluir tanto los determinantes comunes (e.g. comportamientos de salud, estados de morbilidad, toma de medicamentos), además de los determinantes sociales de la salud. Los determinantes sociales de salud (DSS) se definen, como las "condiciones sociales en las cuales viven y trabajan las personas 0 , las características sociales dentro de las cuales la vida tiene lugar. Incluyen tanto las características específicas del contexto social que influyen en la salud como las vías por las cuales las condiciones sociales en que la gente vive se traducen en efectos en salud" (10). La salud oral es una de las patologías cuyos indicadores están fuertemente relacionados con los determinantes sociales, particularmente con factores económicos y sociales (11), que generan fuertes inequidades sociales en salud entre los diferentes grupos de población (12).

La literatura que ha mostrado el impacto que generan las enfermedades bucales, sistémicas y la rehabilitación protésica (7), sobre la CVRSO, sobre pues todas esto afectan la función masticatoria 0 fonética, la apariencia física y la vida social (7). Otros estudios realizados en adultos han mostrado el impacto sobre la CVRSO de las características sociodemográficas (13), como el nivel de escolaridad, ocupación, empleo e ingresos (8).

Este estudio pretende identificar los principales determinantes que pueden influir sobre la CVRSO en personas adultas mayores de un centro diurno del cantón de Desamparados en San José, Costa Rica en 2018. Para esto se han analizado los factores que podrían jugar un rol sobre la CVRSO, desde la salud sistémica, toma de medicamentos, condiciones patológicas bucales como el edentulismo, la xerostomía y los determinantes sociales.

Siendo las patologías orales uno de los principales problemas de salud de las personas adultas mayores en Costa Rica $(14,15)$ y considerando que la calidad de vida es un pilar para garantizar una buena salud, la generación de evidencia científica que permita determinar cuáles son los determinantes de la CVRSO de las personas adultas mayores, podría colaborar para guiar las futuras políticas de promoción de la salud, prevención y atención de las enfermedades.

\section{MATERIALES Y MÉTODOS}

\section{DISEÑO DEL ESTUDIO}

Este proyecto piloto pretende realizar un estudio descriptivo transversal de tipo observacional sobre el estado de salud bucodental de las personas adultas mayores (PAM) en el Centro Diurno Pilar Gamboa de Desamparados. La recolección de datos se realizó entre el 2018 y el 2019. Para este 
estudio se realizó un cuestionario de salud, el cual fue aplicado por encuestadores entrenados. El cuestionario contó con 53 preguntas, las cuales fueron seleccionadas y anteriormente validadas y estandarizadas para esta población en estudios previos realizado en Latinoamérica o en Costa Rica.

\section{CARACTERÍSTICAS DE LA POBLACIÓN}

Los datos provienen de un grupo de personas adultas mayores asistentes (PAM) del Centro Diurno (CD) Pilar Gamboa en Desamparados. Los $C D$ en Costa Rica son instituciones que cuentan en general con apoyo estatal, a los cuales asisten personas de 65 años 0 más, que se encuentran en un proceso de envejecimiento activo. En estos sitios, realizan actividades diurnas sociales, manualidades y actividad física, entre otras. En términos de salud y envejecimiento sano, esta población tendría mejores indicadores de salud general con respecto a la población general adulta mayor costarricense. La población inicial total del CD era de 42 personas. Sin embargo, hubo personas que no pudieron participar por las siguientes razones: muerte, accidentes 0 cirugías que evitaban que pudieran trasladarse al $C D$ y una persona que decidió no participar. La muestra finalmente analizada fue de 32 personas. Se invitó a participar en el estudio a toda la población y se excluyó a aquel adulto que no deseó participar o no firmó el consentimiento informado.

\section{VARIABLES ANALIZADAS}

Variable dependiente: La CVRSO, se midió a través del instrumento GOHAl por sus siglas en inglés General/Geriatric Oral Health Assesment Index. Atchinson y Dolan (16) confeccionaron el GOHAl, que ya ha sido utilizado y validado en poblaciones hispanohablantes (17). El GOHAl se utiliza para medir "problemas funcionales orales reportados por el paciente e impacto psicosocial asociado con enfermedad oral" (18). Consta de 12 preguntas acerca de los problemas en la función bucal y el impacto psicosocial asociado a los problemas orales (18). Las respuestas son registradas usando una escala Likert de 5 puntos $(1=$ siempre, $2=$ a menudo, $3=$ a veces, $4=$ rara vez y $5=$ nunca) (19). La puntuación total corresponde a la suma de las puntuaciones parciales y oscila entre 12 y 60 puntos, siendo el puntaje mayor de 60 puntos un indicador de buena CVRSO y así disminuyendo los valores hasta 12 puntos que muestra una muy pobre CVRSO (19). El GOHAI es considerado el mejor cuestionario para medir el estado de salud bucal subjetivo (19). Además, es corto y ha obtenido suficiente validez en la población adulta mayor.

\section{VARIABLES INDEPENDIENTES}

Dentro de las variables que se analizaron que podrían estar relacionadas con la CVRSO se incluyeron:

\section{Variables sociodemográficas}

La edad ( $<80$ años y $\geq 80$ años) y el sexo (Hombre / Mujer).

\section{Variables socioeconómicas}

Ingresos (dividido en cuartiles Q1: menores ingresos, Q4: mayores ingresos), ocupación (Profesional/No profesional) y escolaridad (Primaria incompleta/Primaria completa).

\section{Variables de morbilidad}

Como hipertensión arterial, diabetes, cáncer, edentulismo total (categorizadas como 0 : No presenta la enfermedad/1: Sí presenta la enfermedad). La xerostomía fue medida como una variable cuantitativa discreta, basada en el instrumento subjetivo Xerostomia Inventory (XI) $(20,21)$. EI XI es un instrumento que utiliza 11 ítems que presentan respuestas graduadas desde el 1 al 5, cuya sumatoria representa la severidad de 
xerostomía crónica (1: menos severidad, 5: mayor severidad). La puntuación total oscila entre $11 \mathrm{y}$ 55 puntos, de acuerdo a la suma de cada uno de los ítems, siendo los valores altos representativos de severos síntomas de xerostomía (20).

\section{Consumo de medicamentos}

Como antihipertensivos, antipsicóticos, analgésicos y antidepresivos (categorizadas como 0 : No consume/1: Sí consume).

\section{Comportamientos de salud}

Como fumado (Nunca/Alguna vez), deporte (Nunca/Alguna vez), consumo de meriendas (Nunca/Alguna vez), consumo de azúcares (Bajo/ Medio/Alto).

\section{ANÁLISIS ESTADÍSTICOS}

Se realizaron análisis bivariados utilizando test no paramétricos, pues no se cumplían con los requisitos de las pruebas paramétricas (distribución normal y homoscedasticidad).

\section{PRUEBA DE MAN WHITNEY (WILCOXON)}

Se utilizó para analizar la relación entre la CVRSO (variable cuantitativa) y la edad, el sexo, la ocupación, la escolaridad, la morbilidad, el fumado, la actividad física, el consumo de medicamentos, consumo de meriendas y consumo de azúcares.

\section{PRUEBA DE KRUSKALL - WALLIS}

Esta prueba no paramétrica se utilizó para analizar la relación entre la CVRSO y los ingresos (dividida en cuartiles).

\section{PRUEBA DE SPEARMAN}

Esta prueba no paramétrica se utilizó para analizar la relación entre la CVRSO y la xerostomía (variable cuantitativa discreta). Todos los análisis fueron realizados utilizando el paquete estadístico STATA 14®.

\section{RESULTADOS}

La tabla 1 muestra la distribución de la variable cuantitativa discreta GOHAl, la variable dependiente de este estudio. Se muestra el total de participantes ( $\mathrm{N}=32)$, su promedio (49.6), su desviación estándar (12.32), el puntaje mínimo obtenido (14) y el puntaje máximo obtenido (60).

La tabla 2 muestra los resultados de las estadísticas bivariadas entre la variable cuantitativa discreta GOHAl y los distintos determinantes analizados en este estudio. La edad se presenta como una variable cualitativa dividida en dos categorías, las personas de 80 años 0 más reportaron una mejor CVRSO (promedio $=50.9$ ) que las personas menores de 80 años (promedio=45.7). Con respecto al sexo, los hombres reportaron una mejor CVRSO (promedio=57.7) comparativamente a las mujeres (promedio=48.8), sin embargo, es importante notar que la cantidad de hombres es muy reducida $(\mathrm{N}=3)$. Para la variable ingresos, se observó una mejor CVRSO a mayor cantidad de ingresos. La ocupación, mostró que los profesionales reportaron una mejor CVRSO (promedio=51.4) en comparación con los no profesionales (promedio=49.1). Para el nivel de escolaridad, las personas con escolaridad mayor a primaria reportaron mejor CVRSO (promedio $=50.0$ ) respecto a aquellas personas con escolaridad igual o menor a primaria (promedio $=48.9$. Con respecto a la morbilidad, las personas con hipertensión reportaron peor CVRSO (promedio=48.4) que las personas que no padecen la enfermedad (promedio=52.6). Con respecto a la diabetes ocurrió lo contrario; las personas con diabetes reportaron una mejor CVRSO (promedio $=50.7$ ) que las personas que no padecen la enfermedad (promedio=49.2). En cuanto a la variable cáncer, las personas que reportaron haber sufrido cáncer presentaron 
en promedio una peor CVRSO (promedio de personas con cáncer $=43.5 /$ Promedio personas sin cáncer=50.0). En general, cualquier estado de morbilidad aumenta en promedio las probabilidades de declararse con una peor CVRSO. Para la variable de edentulismo, las personas edéntulas totales reportaron peor CVRSO (48.0) que aquellas personas sin esta condición (51.0). Con la variable de xerostomía, se reveló una relación estadísticamente significativa entre el puntaje de xerostomía y el puntaje de GOHAl; lo que indica que en promedio un aumento de la sensación de sequedad bucal se relaciona con peores promedios de CVRSO (rho=-0.55, $p=0.0010$ ). En cuanto al consumo de medicamentos, las personas que consumen antidepresivos declararon en promedio una peor CVRSO si se les comparaba con aquellos que no consumen este medicamento (promedio de personas con antidepresivos $=48.3 /$ promedio de personas $\sin$ antidepresivos=54.6). Las personas que consumen antipsicóticos, declaran en promedio una peor CVRSO si se les compara con aquellos que no consumen este medicamento (promedio de personas con antipsicóticos $=50.0 /$ promedio de personas $\sin$ antipsicóticos $=52.6$ ). Las personas que consumen analgésicos, reportan peor CVRSO (promedio=49.8) que aquellas que no los consumen (promedio=53.5). De igual forma, las personas que consumen antihipertensivos reportan peor CVRSO (promedio $=50.2$ ) que aquellas personas que no consumen este medicamento (51.3). En general, el consumo de medicamentos se relaciona con promedios más bajos de CVRSO. Con respecto a los comportamientos de salud, se observa que las personas que han fumado declaran mejor CVRSO (promedio $=56.7$ ) que aquellas que no lo han hecho (promedio=47.61). Para la variable deporte, las personas activas físicamente activas reportan mejor CVRSO (promedio $=51.30$ ) que aquellas personas que no realizan actividad física (promedio=47.98). En cuanto a las meriendas, aquellos que nunca las toman reportan mejor CVRSO que aquellas personas que las realizan alguna vez (promedio de personas que toman merienda $=46.89$ /promedio de personas que no consumen merienda=58.14). Finalmente, se analizaron los datos de la variable consumo de azúcares y se clasifica según el nivel de consumo: bajo (promedio $=48.59$ ), medio (promedio=44.15) y alto (promedio $=50.52$ ). En promedio, se observó una mejor CVRSO entre mayor reporte de consumo de azúcares tenía la persona.

Tabla 1. Distribución de la variable cuantitativa discreta GOHAl.

\begin{tabular}{cccccc}
\hline Variable & N & Promedio & DS & Mínimo & Máximo \\
\hline GOHAl & 32 & 49.6 & 12.3 & 14 & 60 \\
\hline
\end{tabular}


Tabla 2. Estadísticas bivariadas entre el puntaje de GOHAl y los distintos determinantes.

\begin{tabular}{|c|c|c|c|c|}
\hline Variable & N (\%) & Promedio GOHAI & DS & $\mathbf{p}$ \\
\hline \multicolumn{5}{|l|}{ Edad } \\
\hline$<80$ años & $9(28 \%)$ & 45.7 & 13.7 & \multirow[t]{2}{*}{ NS } \\
\hline$>80$ años & 22 (69\%) & 50.9 & 11.9 & \\
\hline Datos faltantes & $1(3 \%)$ & & & \\
\hline \multicolumn{5}{|l|}{ Sexo } \\
\hline Hombre & $3(9 \%)$ & 57.7 & 4.0 & \multirow[t]{2}{*}{ NS } \\
\hline Mujer & $29(90 \%)$ & 48.8 & 12.6 & \\
\hline \multicolumn{5}{|l|}{ Ingresos } \\
\hline Q1 & $8(25 \%)$ & 49.7 & 13.4 & \multirow{4}{*}{$\mathrm{NS}^{2}$} \\
\hline Q2 & $5(16 \%)$ & 51.2 & 6.2 & \\
\hline Q3 & $7(22 \%)$ & 52.0 & 11.6 & \\
\hline Q4 & $5(16 \%)$ & 53.7 & 4.7 & \\
\hline Datos faltantes & $7(22 \%)$ & & & \\
\hline \multicolumn{5}{|l|}{ Ocupación } \\
\hline Profesionales & $7(22 \%)$ & 51.4 & 11.5 & \multirow[t]{2}{*}{$\mathrm{NS}^{1}$} \\
\hline No profesionales & 25 (78\%) & 49.0 & 12.7 & \\
\hline \multicolumn{5}{|l|}{ Escolaridad } \\
\hline$<$ Primaria & $13(40 \%)$ & 48.9 & 12.4 & \multirow[t]{2}{*}{$\mathrm{NS}^{1}$} \\
\hline >Primaria & $19(60 \%)$ & 50.0 & 12.5 & \\
\hline \multicolumn{5}{|l|}{ Hipertensión } \\
\hline 0 & $9(28 \%)$ & 52.6 & 10.6 & \multirow[t]{2}{*}{$\mathrm{NS}^{1}$} \\
\hline 1 & $23(71 \%)$ & 48.4 & 12.9 & \\
\hline \multicolumn{5}{|l|}{ Diabetes } \\
\hline 0 & 24 (75\%) & 49.2 & 13.2 & \multirow[t]{2}{*}{$\mathrm{NS}^{1}$} \\
\hline 1 & $8(25 \%)$ & 50.7 & 10.6 & \\
\hline \multicolumn{5}{|l|}{ Cáncer } \\
\hline 0 & 30 (93\%) & 50.0 & 12.4 & \multirow[t]{2}{*}{$\mathrm{NS}^{1}$} \\
\hline 1 & $2(6 \%)$ & 43.5 & 12.0 & \\
\hline \multicolumn{5}{|l|}{ Morbilidad } \\
\hline 0 & $8(25 \%)$ & 53.1 & 11.2 & \multirow[t]{2}{*}{ NS1 } \\
\hline 1 & 24 (75\%) & 48.4 & 12.7 & \\
\hline \multicolumn{5}{|l|}{$\begin{array}{l}\text { Enfermedad } \\
\text { autoreportada }\end{array}$} \\
\hline 0 & $7(21 \%)$ & 55.9 & 4.6 & \\
\hline 1 & 24 (75\%) & 47.3 & 13.3 & NS1 \\
\hline Datos faltantes & $1(3 \%)$ & & & \\
\hline \multicolumn{5}{|l|}{ Edentulismo total } \\
\hline 0 & 17 (53\%) & 51.0 & 11.1 & \multirow[t]{2}{*}{$\mathrm{NS}^{1}$} \\
\hline 1 & 15 (46\%) & 48.0 & 13.8 & \\
\hline \multicolumn{5}{|l|}{ Xerostomía } \\
\hline Spearman's rho & $32(100 \%)$ & -0.5526 & 0.0010 & $S^{\star 3}$ \\
\hline
\end{tabular}




\begin{tabular}{|c|c|c|c|c|}
\hline Variable & N (\%) & Promedio GOHAI & DS & $p$ \\
\hline \multicolumn{5}{|l|}{ Antidepresivos } \\
\hline 0 & $19(59 \%)$ & 54.6 & 6.7 & NS1 \\
\hline 1 & $10(31 \%)$ & 48.3 & 11.1 & \\
\hline Datos faltantes & $3(9 \%)$ & & & \\
\hline \multicolumn{5}{|l|}{ Antipsicóticos } \\
\hline 0 & $26(8 \%)$ & 52.6 & 9.3 & NS1 \\
\hline 1 & $2(6 \%)$ & 50.0 & 4.3 & \\
\hline Datos faltantes & $4(1 \%)$ & & & \\
\hline \multicolumn{5}{|l|}{ Analgésicos } \\
\hline 0 & $17(53 \%)$ & 53.9 & 7.3 & $\mathrm{NS}^{1}$ \\
\hline 1 & $12(37 \%)$ & 49.8 & 11.4 & \\
\hline Datos faltantes & $3(9 \%)$ & & & \\
\hline \multicolumn{5}{|l|}{ Antihipertensivo } \\
\hline 0 & $8(25 \%)$ & 51.3 & 11.5 & $\mathrm{NS}^{1}$ \\
\hline 1 & $22(68 \%)$ & 50.2 & 10.7 & \\
\hline Datos faltantes & $2(6 \%)$ & & & \\
\hline \multicolumn{5}{|l|}{ Fumado } \\
\hline Nunca & $25(78 \%)$ & 47.6 & 13.2 & $\mathrm{NS}^{1}$ \\
\hline Alguna vez & $7(21 \%)$ & 56.7 & 3.8 & \\
\hline \multicolumn{5}{|l|}{ Deporte } \\
\hline Nunca & 19 (59\%) & 48.0 & 13.3 & \\
\hline Alguna vez & $12(37 \%)$ & 51.3 & 11.0 & $\mathrm{NS}^{1}$ \\
\hline Datos faltantes & $1(3 \%)$ & & & \\
\hline \multicolumn{5}{|l|}{ Merienda } \\
\hline Nunca & $5(15 \%)$ & 58.1 & 1.8 & NS1 \\
\hline Alguna vez & $24(75 \%)$ & 46.9 & 13.1 & \\
\hline Datos faltantes & $3(9 \%)$ & & & \\
\hline \multicolumn{5}{|c|}{ Consumo de azúcares } \\
\hline Bajo & $8(25 \%)$ & 48.6 & 12.2 & $\mathrm{NS}^{1}$ \\
\hline Medio & $4(12.5 \%)$ & 44.2 & 16.8 & \\
\hline Alto & 15 (46.87\%) & 50.5 & 13.5 & \\
\hline Datos faltantes & 5 (15.62\%) & & & \\
\hline
\end{tabular}

NS: Estadísticamente No Significativo

$S^{*}$ : Estadísiticamente significativo

1Test no paramétrico de Mann-Whitney o Test de Wilcoxon

2 Test no paramétrico de Kruskall-Wallis

3 Test no paramétrico de Spearman 
DISCUSIÓN

Este estudio revela que existen diferencias de promedio al comparar la calidad de vida relacionada con la salud oral y los determinantes demográficos, socioeconómicos, de morbilidad, toma de medicamentos y comportamientos de salud. Los mayores de 80 años, las mujeres, aquellos con niveles superiores de escolaridad, de ingresos altos, sin edentulismo, con niveles bajos de xerostomía, las personas sin enfermedades, que no toman medicamentos, que no fuman, hacen deporte, que no meriendan y que consumen moderadamente azúcares, son aquellos que reportan una mejor CVRSO comparados a sus contrapartes. Sin embargo, no se pudo poner en evidencia diferencias estadísticamente significativas (excepto por la relación entre CVRSO y la xerostomía), debido al reducido tamaño de la muestra. Al no existir ningún estudio similar realizado en el país, los resultados obtenidos no pueden ser comparados, no obstante, al ser un estudio piloto, permitirán abrir paso a futuras investigaciones.

Desde el análisis estadístico realizado, es importante destacar que se observó una diferencia en la distribución entre la CVRSO y sexo y edad. Esto expone que en las mujeres hay una tendencia a una percepción de CVRSO más baja que en los hombres, lo que coincide con lo referido por De La Fuente y colaboradores quienes reportan que en los hombres se observó un menor impacto de su salud bucal sobre su CVRSO (22). Tal situación puede ser explicada, porque las mujeres se preocupan más por su estado de salud. No obstante, esto no necesariamente refleja el que los hombres gocen de mejor salud, situación que puede derivarse de la influencia cultural y de género (23). En cuanto a la variable edad, las personas mayores de 80 años reportan una CVRSO superior, lo cual difiere con Io analizado por Duque et al., quienes concluyen que a mayor edad se cuenta con menor CVRSO; posiblemente debido al hecho que a medida que avanza la edad, las personas perciben un deterioro progresivo de su CVRSO (24).

Otros factores condicionantes de la CVRSO y su relación con la salud bucal en la población estudiada incluyen el nivel educativo, ingresos y ocupación. Los resultados obtenidos al analizar estas variables no son estadísticamente significativos, pero todos apuntan que, a menor ingreso económico, menor escolaridad y el no ser profesional se relacionan de manera directa con una percepción de CVRSO relacionada con salud oral menor. Esto coincide con los resultados obtenidos por Duque y colaboradores (24) , lo cual puede ser explicado por barreras culturales propias del individuo que podrían en algún momento determinado hacer más difícil la asimilación de información relacionada con salud bucal, lo cual, en muchas ocasiones, se suma al proceso de envejecimiento (25).

También se analizó, la relación de la CVRSO y estados de morbilidad, donde se incluyen patologías como hipertensión arterial, diabetes, cáncer, edentulismo y xerostomía. En general, cualquier estado de morbilidad aumenta en promedio las probabilidades de declararse con una menor CVRSO relacionada con la salud bucal, si se comparan con el grupo de personas sin morbilidades. Es importante mencionar que, la variable xerostomía sí resulta con significancia estadística, donde en promedio, un aumento de la sensación de sequedad bucal se relaciona con promedios de CVRSO relacionada con la salud más bajos. Este resultado concuerda con los hallazgos de Hahnel y colaboradores, quienes reportan que la CVRSO en pacientes de edad avanzada disminuye en función de una mayor percepción subjetiva de la boca seca pero no del flujo salival estimulado (26). La xerostomía es más frecuente en la población de edad avanzada, principalmente debido al mayor uso de medicamentos. Además, se ha informado que los pacientes con dentadura 
postiza completa con xerostomía tienen puntos de dolor más intensos que los pacientes con flujo salival normal, aspecto negativo que se asocian de manera directa con una entendible percepción de CVRSO relacionada con salud oral inferior $(27,28)$.

Otra de las variables analizadas en este estudio es el consumo de medicamentos. En general, el consumo de medicamentos se relaciona con promedios más bajos de CVRSO relacionada con la salud bucal. Se incluyeron en este estudio medicamentos como antihipertensivos, antipsicóticos, analgésicos y antidepresivos, I0 cuales no resultan de significancia estadística.

Finalmente, al analizar la relación entre CVRSO relacionada con salud oral y comportamientos de salud, donde se incluye el fumado, realización de actividad física, consumo de azúcar y frecuencia de consumo de alimentos, se encontró que los que alguna vez fumaron declaran en promedio una CVRSO superior. Esto no concuerda con lo analizado por Díaz y colaboradores quienes reportan una baja percepción en la CVRSO en los adultos mayores que presentan el hábito de fumado y lo relacionan a que el tabaquismo constituye un factor de riesgo para patologías bucales como la periodontitis, la cual se vincula con pérdida dental y por lo tanto a una peor CVRSO (29). Esta discrepancia que se encuentra en nuestro estudio se podría deber a que hasta mediados del pasado siglo XX, en Costa Rica la costumbre de fumar tabaco era considerada como un hábito placentero e incluso distinguido, inocuo y socialmente bien visto, 10 cual se relaciona estrechamente con la parte socioeconómica, por lo tanto, con la percepción de una mejor CVRSO (30).

En cuanto a la realización de actividad física y consumo de azúcar, no se pudo poner en evidencia la existencia de una relación estadísticamente significativa. Pero este análisis concluye que, a mayor realización de deporte y consumo de azúcar, mejor CVRSO relacionada con salud oral.
Por su parte, las personas que consumen más alimentos durante el día, son aquellas que tienen en promedio inferior CVRSO relacionada con la salud bucal. En nuestro estudio es posible que se encuentre resultados contradictorios por la fuerte relación que se observa entre la presencia de xerostomía y el consumo frecuente de alimentos. Es posible que este resultado se encuentre confundido con el hecho que las personas con xerostomía son aquellas que, para mitigarla, consumen más alimentos durante el día.

Entre las limitaciones que se encontraron en este estudio se puede mencionar el tamaño de la muestra, el cual al ser tan pequeño no permite que los resultados sean considerados con significancia estadística. Sumado a esto, no se realizó un análisis multivariado para relacionar las variables entre sí. Esto hubiera permitido observar el efecto propio de cada variable sobre la CVRSO relacionada a la salud oral. También se debe tomar en cuenta que la composición de la muestra no es homogénea, esto quiere decir que al no contar con la misma cantidad, por ejemplo de hombres y mujeres, no se permitió dar a conocer la autopercepción que tiene el adulto mayor abordado desde ambos sexos por igual, además, se reconoce que el adulto mayor generalmente no proporciona en forma espontánea toda la información necesaria, debido a que creen que sus problemas forman parte del proceso de envejecimiento o sienten desconfianza al momento de contestar la entrevista.

A pesar de estas limitaciones, se debe incluir como fortalezas que éste estudio se constituye como pionero en el país al incluir estas variables en la población adulta mayor. Es importante mencionar que, al ser un estudio piloto, los resultados obtenidos permitirán hacer las estimaciones adecuadas para un cálculo de muestra que permita poner en evidencia relaciones que se consideren significativas estadísticamente, y con esto, validar un protocolo de investigación que busca mejorar la comprensión de los elementos sociales 
potencialmente relacionados con la salud general y bucodental para una población adulta mayor costarricense asistente a centros diurnos.

\section{CONCLUSIÓN}

Este estudio piloto permitió relevar las principales variables que podrían estar relacionada con la calidad de vida relacionada con la salud oral en personas adultas mayores en proceso de envejecimiento activo. Este ha permitido la obtención de informaciones valiosas que permiten discutir de mejor manera con los tomadores de decisiones en salud pública sobre las principales necesidades de salud bucodental de las personas adultas mayores en Costa Rica, así como bridar apoyo financiero para futuras investigaciones representativas de este grupo de edad.

\section{AGRADECIMIENTOS}

Los autores desean agradecer a los miembros de la Junta Directiva del Centro Diurno Pilar Gamboa de Desamparados, por darnos la oportunidad de realizar este estudio piloto en sus instalaciones. Agradecemos asimismo a todos (as) los (as) participantes, por contribuir de manera sustancial con sus datos e información de salud tan valiosa para la investigación científica. Agradecemos finalmente a los voluntarios que contribuyeron en el desarrollo de la recolección de datos.

\section{REFERENCIAS}

1. Brenes G., Araya O., Jinesta K., Mora S., Fuentes E., González M.E., etal. Estimaciones y Proyecciones de Población por sexo y edad 1950-2050. San José, Costa Rica; 2013.

2. Cesare M. Di. El perfil epidemiológico de América Latina y el Caribe: desafíos, límites y acciones. Santiago de Chile; 2011.

3. Barboza Solis C., Fantin R., Kelly-Irving M., Delpierre C. Physiological wear-andtear and later subjective health in mid-life: Findings from the 1958 British birth cohort. Psychoneuroendocrinology. 2016; 74: 24-33.

4. Gallo V., Mackenbach J.P., Ezzati M., Menvielle G., Kunst A.E., Rohrmann S., et al. Social inequalities and mortality in europe - results from a large multi-national cohort. PLoS One. 2012; 7 (7): 1-11.

5. Engel G.L. The need for a new medical model: a challenge for biomedicine. Science. 1977;196 (4286):129-36.

6. Ageing H. Joint Report on Operational Definition of Healthy Ageing. 2015; 6(633666).

7. Degrandi V., Bentancourt M., Fabruccini A., Fuentes F. Assessment of the Impact on Quality of Life in Adult Patients Treated With New Complete Removable Dentures. Odontoestomatologia. 2017; 19 (29).

8. Díaz-Cárdenas S., Meisser-Vidal M.A., TiradoAmador L.R., Fortich-Mesa N., Tapias-Torrado 
L., González-Martínez F.D. Impacto de Salud Oral sobre Calidad de Vida en Adultos. Int J Odontostomatol. 2017; 11: 5-11.

9. Watt R.G., Listl S., Peres M., Heilmann A. Social inequalities in oral health: from evidence to action. London: University College London; 2015.

10. OrganizaciónMundialdelaSalud.Determinantes Sociales de la Salud [Internet]. Available from: https://www.who.int/topics/social_determinants/ es/. (Accessed in March 2019).

11. Kwan S., Petersen P.E. Equity, social determinant and public health programmes. Blas E., Kurup A.S., editors. Geneva; 2010. 293 p.

12. Sabbah W., Tsakos G., Chandola T., Sheiham A., Watt R.G. Social gradients in oral and general health. J Dent Res. 2007; 86 (10): 992-6.

13. Alzate-Urrea S., Agudelo-Suárez A.A., López-Vergel F., López-Orozco C., EspinosaHerrera É., Posada-López A., et al. Calidad de vida y salud bucal: Perspectiva de adultos mayores atendidos en la red hospitalaria pública de Medellín, Colombia. Rev Gerenc y Polit Salud. 2015; 14 (29): 83-96.

14. Barboza Solís C., Fantin R. The Role of Socioeconomic Position in Determining Tooth Loss in Elderly Costa Rican: Findings from the CRELES Cohort. Vol. 19, Odovtos International Journal of Dental Sciences . scielo; 2017. p. 79-94.

15. Fantin R., Delpierre C., Kelly-Irving M., Barboza Solís C. Early socioeconomic conditions and severe tooth loss in middle-aged Costa Ricans. Community Dent Oral Epidemiol. 2018; 46 (2): 178-84.

16. Atchison K.A., Dolan T.A. Development of the Geriatric Oral Health Assessment Index. J Dent Educ. 1990; 54 (11): 680-7.

17. Salazar Díaz O.A. Validación en Chile del cuestionario GOHAI y Xerostomía Inventory (XI) en adultos mayores. Universidad de Chile; 2010.
18. Locker D., Allen F. What do measures of "oral health-related quality of life" measure? Community Dent Oral Epidemiol. 2007; 35 (6): 401-11.

19. Locker D. Concepts of oral health, disease and the quality of life. In: Slade GD, editor. Measuring oral health and quality of life. Chapel Hill; 1996. p. 11-22.

20. Thomson W.M., Chalmers J.M., Spencer A.J., Williams S.M. The Xerostomia Inventory: a multi-item approach to measuring dry mouth. Community Dent Health. 1999;16 (1):12-7.

21. Serrano C., Farina M.P., Perez C., Fernandez M., Forman K., Carrasco M. Translation and validation of a Spanish version of the xerostomia inventory. Gerodontology. 2016; 33 (4): 506-12.

22. De la Fuente J., Sumano O., Sifuentes M.C., Zelocuatecatl A. Impacto de la salud bucal en la calidad de vida de adultos mayores demandantes de atención dental. Univ Odontol. 2010; 29 (63): 83-92.

23. Jiménez Pérez J., Esquivel Hernández R.I., González-Celis Rangel A.L. La percepción de salud bucal como medida de la calidad de vida en ancianos. Rev ADM. 2003; 60 (1): 19-24.

24. Duque-Duque V.E., Tamayo-Castrillón J., Echeverri-Cadavid P.A., Gutiérrez-Osorio A.Y., Sepúlveda-Correa D., Giraldo-Ramírez O., et al. Oral health related quality of life in older adults assisting "IPS Universitaria" of Medellin and associated factors. CES Odontol. 2013; 26 (1): 10-23.

25. Olmedo M.M., Ramírez J., Sánchez M.A., Barrilao G, C. V. Envejecimiento y calidad de vida en una sociedad multicultural. Scientia. 2010; 15 (2): 135-52.

26. Hahnel S., Schwarz S., Zeman F., Schaefer L., Behr M. Prevalence of xerostomia and hyposalivation and their association with quality of life in elderly patients in dependence on dental status and prosthetic 
rehabilitation: A pilot study. J Dent. 2014; 42 (6): 664-70.

27. Rebelo M.A.B., Cardoso E.M., Robinson P.G., Vettore M.V. Demographics, social position, dental status and oral health-related quality of life in community-dwelling older adults. Qual Life Res. 2015; 25 (7): 1735-42.

28. Sreebny L.M., Schwartz S.S. A reference guide to drugs and dry mouth--2nd edition. Gerodontology. 1997;14 (1): 33-47.
29. Díaz C. V., Pérez N.M., Ferreira M.I., Sanabria D.A., Torres C.D., Araujo A., et al. Autopercepción de la calidad de vida relacionada con salud oral en adultos mayores residentes en albergues estatales del área metropolitana, Paraguay. Rev Odontológica Latinoam. 2015; 7 (1): 23-31.

30. Acuña V. H. Historia económica del tabaco en Costa Rica: Época ColoniaI. San José, Costa Rica; 1976. 279-392 p. 


\section{Anexo}

Teléfono: 2511-5449 / Fax 2224-9223 / Correo electrónico:odontologia.socialucr@gmail.com

\section{Departamento de Odontología Social \\ Sección de Promoción de la Salud Oral y Prevención \\ Proyecto de investigación Centro Diurno Pilar Gamboa \\ \# Expediente:}

\section{Cuestionario GOHAI de calidad de vida relacionada a la salud oral}

\begin{tabular}{|c|c|c|c|c|c|}
\hline Con qué frecuencia en los últimos 3 meses... & Siempre & A menudo & $\begin{array}{c}\text { A } \\
\text { veces }\end{array}$ & Rara vez & Nunca \\
\hline $\begin{array}{l}\text { 1.- ¿Cuántas veces limitó la clase o cantidad de } \\
\text { alimentos que come debido a problemas con sus dientes } \\
\text { o prótesis dentales? }\end{array}$ & 1 & 2 & 3 & 4 & 5 \\
\hline $\begin{array}{l}\text { 2.- ¿Con qué frecuencia no pudo tragar bien o } \\
\text { cómodamente? }\end{array}$ & 1 & 2 & 3 & 4 & 5 \\
\hline $\begin{array}{l}\text { 3.- ¿Cuántas veces sus dientes o prótesis dentales le } \\
\text { impidieron hablar de la manera que usted quería? }\end{array}$ & 1 & 2 & 3 & 4 & 5 \\
\hline $\begin{array}{l}\text { 4.-¿Cuántas veces sintió molestias al tratar de comer lo } \\
\text { que usted quería? }\end{array}$ & 1 & 2 & 3 & 4 & 5 \\
\hline $\begin{array}{l}\text { 5.- ¿Cuántas veces evitó estar en contacto con personas } \\
\text { debido a la condición de sus dientes o prótesis dentales? }\end{array}$ & 1 & 2 & 3 & 4 & 5 \\
\hline $\begin{array}{l}\text { 6.- ¿Cuántas veces estuvo insatisfecho o no contento } \\
\text { con la apariencia de sus dientes, encías o prótesis } \\
\text { dentales? }\end{array}$ & 1 & 2 & 3 & 4 & 5 \\
\hline $\begin{array}{l}\text { 7.- ¿Con qué frecuencia estuvo preocupado o } \\
\text { intranquilo por problemas con sus dientes, encías o } \\
\text { prótesis dentales? }\end{array}$ & 1 & 2 & 3 & 4 & 5 \\
\hline $\begin{array}{l}\text { 8.- ¿Con qué frecuencia se sintió nervioso o consciente } \\
\text { debido a problemas con sus dientes, encías o prótesis } \\
\text { dentales? }\end{array}$ & 1 & 2 & 3 & 4 & 5 \\
\hline $\begin{array}{l}\text { 9.- ¿Con qué frecuencia se sintió incómodo al comer } \\
\text { frente a otras personas debido a problemas con sus } \\
\text { dientes, encías o prótesis dentales? }\end{array}$ & 1 & 2 & 3 & 4 & 5 \\
\hline $\begin{array}{l}\text { 10.- ¿Con qué frecuencia evitó reírse o sonreírse debido } \\
\text { a dientes o encías } \\
\text { inatractivas? }\end{array}$ & 1 & 2 & 3 & 4 & 5 \\
\hline $\begin{array}{l}\text { 11.- ¿Con qué frecuencia ha tenido dolor o molestias } \\
\text { alrededor de la boca? }\end{array}$ & 1 & 2 & 3 & 4 & 5 \\
\hline $\begin{array}{l}\text { 12.- ¿Con qué frecuencia estuvieron sus dientes o } \\
\text { encías sensibles a los alimentos calientes, fríos o } \\
\text { dulces? }\end{array}$ & 1 & 2 & 3 & 4 & 5 \\
\hline
\end{tabular}

Cuestionario Índice de Salud Oral Geriátrico (GOHAI). Traducido desde Atchinson y Dolan 1990 (1) y validado en población adulta mayor chilena por Salazar (2) en el año 2010.

1. Atchison KA, Dolan TA. Development of the Geriatric Oral Health Assessment Index. J Dent Educ. United States; 1990 Nov;54(11):680-7.

2. Salazar, O. Validación en Chile del cuestionario GOHAI y Xerostomía Inventory ( XI ) en adultos mayores. Universidad de Chile; 2010.
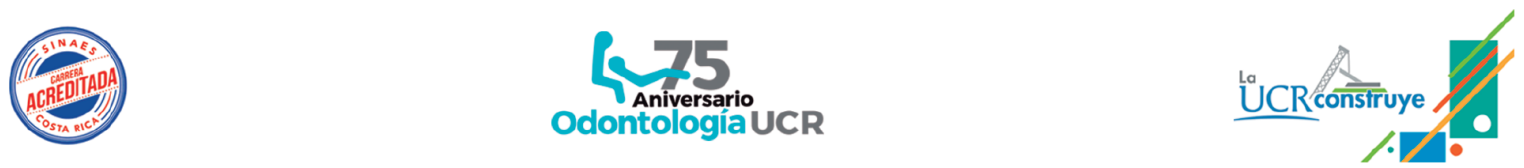